\title{
ON FUNDAMENTAL GROUPS OF POSITIVELY CURVED MANIFOLDS WITH LOCAL TORUS ACTIONS*
}

\author{
XIAOCHUN RONG ${ }^{\dagger}$
}

\begin{abstract}
In this paper, we study the fundamental groups of closed manifolds of positive sectional curvature which admit compatible local isometric torus $T^{k}$-actions. We explore relations between basic properties of an isometric $T^{k}$-action and the structure of the fundamental group of $M$. Using these relations, we prove several results on the fundamental groups.
\end{abstract}

Key words. Positive sectional curvature, isometric torus action and fundamental groups

AMS subject classifications. 53C21

0. Introduction. Let $M$ denote a manifold. Recall that a $\pi_{1}$-invariant torus $T^{k}$-action on $M$ is defined by an effective $T^{k}$-action on its a universal covering space $\tilde{M}$ that extends to the action by the semi-direct product, $T^{k} \ltimes_{\rho} \pi_{1}(M)$, where $\rho$ is a homomorphism from the fundamental group $\pi_{1}(M)$ to the automorphism group of $T^{k}$ ([Ro2]). Clearly, $\rho$ is a trivial map (or equivalently, the $T^{k}$-action commutes with the deck transformations) if and only if the $T^{k}$-action on $\tilde{M}$ is the lifting of a $T^{k}$-action on $M$. We call the projection on $M$ of a $T^{k}$-orbit in $\tilde{M}$ an orbit of the $\pi_{1}$-invariant $T^{k}$-action.

Let's make a convention once and for all: Any $T^{k}$-action considered in this paper is assumed to be effective, unless mentioned otherwise.

In the case where $\pi_{1}(M)$ is finite, a $\pi_{1}$-invariant $T^{k}$-action is equivalent to the notion of a pure F-structure, introduced by Cheeger-Gromov in the study of collapsing Riemannian manifolds with bounded curvature and diameter ([CG1,2]). This includes, up to a finite exceptions, the class of pinched positive sectional curvature manifolds ([Ro1,2]).

In this paper, we study the fundamental group of a positively curved manifold $M$ which admits a $\pi_{1}$-invariant isometric $T^{k}$-action ([Ro1,2]). By the classical Synge theorem, we implicitly assume that the dimension of $M$ is odd. We will explore relations between properties of a $T^{k}$-action and the structure of the fundamental group of $M$ (see Theorems $\mathrm{A}$ and $\mathrm{C}$ ). Using these relations, we prove several results on the fundamental groups, including generalizations of the main results in [Ro1,2] (see Theorems B and D).

We point out that our study is closely related to Grove's proposal on the classification of positively curved manifolds with large isometry group (cf. [Gro] and references within, [FMR], [FR1,2], [Wi]). In the non-simply connected situation, the first step toward a classification is to classify the fundamental groups (see [FR4], [Ro4]).

One basic tool in this paper is the following Synge-type result.

THEOREM A. Let $M$ be a closed manifold of positive sectional curvature on which a torus $T^{k}(k \geq 1)$ acts isometrically. If $\phi$ is an isometry on $M$ commuting with the

* Received September 25, 2005; accepted for publication November 10, 2005.

${ }^{\dagger}$ Current Address: Mathematics Department, Rutgers University New Brunswick, NJ 08903 USA (rong@math.rutgers.edu); Mathematics Department, Capital Normal University, Beijing, P.R.C. Supported partially by NSF Grant DMS 0203164 and by a research fund from Capital normal university. 
$T^{k}$-action, then $\phi$ preserves some $T^{k}$-orbit which is a circle.

The existence of a circle $T^{k}$-orbit essentially follows Berger's vanishing theorem, see [GS], [Ro1] and [Su].

We first give a consequence of Theorem A.

COROLlary 0.1. Let $M$ be a closed manifold of positive sectional curvature on which $T^{k}$ acts isometrically. If a principle $T^{k}$-orbit contains a homotopy nontrivial loop (in $M$ ), then the fundamental group $\pi_{1}(M)$ is not isomorphic to $\mathbb{Z}_{p} \oplus \mathbb{Z}_{p}$ for any prime $p$.

Corollary 0.1 provides a sufficient condition for a partial positive answer to the well-known question of S. S. Chern ([Ch], [Ya]): for a closed manifold $M$ of positive sectional curvature, is every abelian subgroup of $\pi_{1}(M)$ cyclic? Note that a negative answer was recently found in dimensions 7 and 13 ([Ba], [GS], [Sh]).

Theorem A is also a crucial ingredient in the proofs of Theorems B-D below.

We call a normal cyclic subgroup of a group maximal, if it is not properly contained in any normal cyclic subgroup (here we allow a trivial maximal cyclic group).

Theorem B (Maximal normal Cyclic subgroups). Let $M$ be a closed $n$ manifold of positive sectional curvature which admits a $\pi_{1}$-invariant isometric $T^{k}$ action. If $C$ is a maximal normal cyclic subgroup of $\pi_{1}(M)$, then its index, $\left[\pi_{1}(M)\right.$ : $C] \leq w(n)$, a constant depending only on $n$.

Note that Theorem B does not hold if one removes the requirement of "normal" without imposing further restrictions ([Sh]). The existence of a nontrivial normal cyclic subgroup (when $\left|\pi_{1}(M)\right|>w(n)$ ) is closely related to the existence of a nontrivial normal solvable subgroup; see [FY] (Theorem 2.9).

Theorem B generalizes the main result of [Ro1], which asserts (under the same assumptions of Theorem B) that $\pi_{1}(M)$ has some cyclic subgroup with index less than $w(n)$. Because Theorem B applies to any maximal normal cyclic subgroup, we have:

COROLlary 0.2. Let $M$ be a closed n-manifold of positive sectional curvature on which $T^{k}$ acts isometrically. Then $\pi_{1}(M)$ is not isomorphic to $\mathbb{Z}_{p} \oplus \mathbb{Z}_{p^{r}}$ for pr $\geq w(n)$, where $p$ is a prime.

We remark that Corollary 0.2 may apply to any prime $p$. For $r=1$, Corollary 0.2 says that $\pi_{1}(M)$ cannot be isomorphic to $\mathbb{Z}_{p} \oplus \mathbb{Z}_{p}$ for any prime $p \geq w(n)$ ([Ro1]). On the other hand, Corollary 0.2 may not be true if $p r$ is small (e.g., $p r=2,3$, see ([Sh], [GS]).

Corollary 0.3. Let $M$ be a closed n-manifold of positive sectional curvature which admits a $\pi_{1}$-invariant isometric $T^{k}$-action. Then $\pi_{1}(M)$ has a normal cyclic subgroup of index $\leq w(n)$. In particular, if $\pi_{1}(M)$ has only the trivial normal cyclic subgroup, then $\left|\pi_{1}(M)\right| \leq w(n)$.

Because the simple group $A_{5}$ can act freely and isometrically on some Eschenburg 7-manifold of positive sectional curvature on which a circle acts isometrically ([Sh]), we see that $w(7) \geq 50$.

A natural question concerning Theorem $\mathrm{B}$ is to find a criterion by which a fundamental group is cyclic. We give the following answer:

THeOREM C. Let $M$ be a closed n-manifold of positive sectional curvature on 
which $T^{k}$ acts isometrically. If there is no finite isotropy group, then $\pi_{1}(M)$ is cyclic.

Observe that in the case of $k=1$, the condition amounts to a semi-free isometric $T^{1}$-action. If the $T^{1}$-action is free, then Theorem $\mathrm{C}$ is seen from the homotopy exact sequence of the $T^{1}$-fibration and the Synge theorem applied to the orbit space.

By the symmetry rank restriction ([GS]) and by the Frankel's theorem ([Fr]), Theorem $\mathrm{C}$ yields

THEOREM D. Let $M$ be a closed n-manifold of positive sectional curvature on which $T^{k}$ acts isometrically. If $k>\frac{n+1}{4}$, then $\pi_{1}(M)$ is cyclic.

Theorem D was first obtained in [Ro4] for " $k \geq \frac{n+6}{4}$ " (cf. [GS]), and was improved in [Wi] to " $k \geq \frac{n}{4}+1$ ". For $n \neq 3 \bmod 4$, " $k>\frac{n+1}{4}$ " is equivalent to " $k \geq \frac{n}{4}$ ". Theorem $\mathrm{D}$ is optimal for $n \equiv 3 \bmod 4$ (e.g. any finite group of $S U(2)$ can act freely and isometrically on a homogeneous sphere $\left.S^{4 m+3}=S p(m+1) / S p(m)\right)$. For a recent development on the fundamental groups of positively curved manifolds with large symmetry rank, see [RW1,2] and [FRW].

We conclude the introduction with a little prospective on the above results.

The fundamental group of a closed manifold of positive Ricci is finite ([My]), and any finite group can arise as the fundamental group of such a manifold. However, whether or not the latter holds for positive sectional curvature has been a long-standing problem in Riemannian geometry. By the Synge theorem ([Sy]), this problem is open in odd dimensions.

One obstacle is the lack of examples; except in dimensions 7 and 13 ([Ba], [GS], [Sh], [GSZ]), all examples of the fundamental groups are those acting freely and isometrically on round spheres. On the other hand, not a conjectured general obstruction is known.

Our results on the fundamental groups in this paper may be considered as a step toward an answer to the above converse question. In particular, our results may shed light on the following problems.

Conjecture 0.4 . Let $M$ be a closed $n$-manifold of positive sectional curvature. Then every maximal normal cyclic subgroup of $\pi_{1}(M)$ has index less than a constant depending only on $n$.

Theorem B partially verifies Conjecture 0.4. The example of the spherical space forms ([Wo]) shows that the dependence on $n$ of the index bound is the best one may hope for. Note that Conjecture 0.4 implies the almost cyclicity conjecture in [Ro1].

The following question is partially motivated by Corollary 0.3 .

Problem 0.5. Does there exist a universal constant $C>0$ such that if $M$ admits a metric of positive sectional curvature, then $\pi_{1}(M)$ has either order less than $C$ or not a simple group?

Note that a positive answer to Problem 0.5 would imply, in particular, that the alternating group $A_{m}$, for $m>C$, cannot be the fundamental group of any positively curved manifold (compare to [Sh]).

The rest of the paper is organized as follows: In Section 1, we will prove Theorem A. In Section 2, we will prove Theorem B. The proofs of Theorems C and D are given in Section 3. 
Acknowledgment. It is a pleasure to thank Chang-Wan Kim, Xiaole Su and Yusheng Wang for some comments on a preliminary version of this paper, and to thank Krishnan Shankar for a helpful conversation.

\section{Proof of Theorem A.}

a. Isotropy groups and orbit spaces. Consider a compact Lie group $G$ acting isometrically on a manifold $M$. Let $M^{*}=M / G$ denote the orbit space. The quotient metric $d^{*}$ on $M^{*}$ is defined as follows: for any $x^{*}, y^{*} \in M^{*}$, let $x, y \in M$ such that $p(x)=x^{*}$ and $p(y)=y^{*}$. Then $d^{*}\left(x^{*}, y^{*}\right) \stackrel{\text { def }}{=} d(G(x), G(y))$, where $G(x)$ denotes the orbit at $x$. Clearly, the orbit projection, $p: M \rightarrow M^{*}$, is distance non-increasing. Let $\sigma$ be any geodesic jointing $G(x)$ and $G(y)$ such that $d(G(x), G(y))=\operatorname{length}(\sigma)$. Then length $(\sigma)=d\left(x^{*}, y^{*}\right)=\operatorname{length}\left(\sigma^{*}\right)$, where $\sigma^{*}=p(\sigma)$. Hence, the distance in $M^{*}$ is realized by the length of some curve in $M^{*}$.

In the proof of Theorem A, we will use the following simple fact $([\mathrm{Kl}])$ :

LEMMA 1.1. Assume that a compact Lie group $G$ acts isometrically on $M$. If $\gamma(t)$ is a minimal geodesic from $G(\gamma(0))$ to $G(\gamma(1))$, then for all $0<t<1$, the isotropy group at $\gamma(t), G_{\gamma(t)}$, is a subgroup of both $G_{\gamma(0)}$ and $G_{\gamma(1)}$.

We will also give a simple proof (compare to $[\mathrm{Kl}]$ ).

Proof of Lemma 1.1. Let $x_{0}=\gamma(0), x_{1}=\gamma(1)$, and $x=\gamma(t), 0<t<1$. We need to show that $G_{x}\left(x_{0}\right)=x_{0}$ and $G_{x}\left(x_{1}\right)=x_{1}$. We argue by contradiction. Assume $\alpha \in G_{x}$ such that $\alpha\left(x_{0}\right) \neq x_{0}$. Then there is a geodesic triangle whose two sides are $\alpha\left(\left.\gamma\right|_{[0, t]}\right)$ and $\left.\gamma(t)\right|_{[t, 1]}$. Because there is a corner at the vertex $x$, $d\left(\alpha\left(x_{0}\right), x_{1}\right)<d\left(\alpha\left(x_{0}\right), x\right)+d\left(x, x_{1}\right)=d\left(x_{0}, x\right)+d\left(x, x_{1}\right)=d\left(x_{0}, x_{1}\right)=d(G(x), G(y))$, a contradiction

Let $M_{0}$ denote the union of principle $T^{k}$-orbits. Then $M_{0}$ is an open submanifold. By Lemma 1.1, $M_{0}^{*}=M_{0} / T^{k}$ is an open manifold which is also convex.

b. Positive curvature and symmetry rank. The following result is a fundamental fact about an isometric $T^{k}$-action on a closed manifold of positive sectional curvature:

THEOREM 1.2. Let a torus $T^{k}$ act isometrically on a closed manifold $M$ of positive sectional curvature.

(1.2.1) (Berger) If $\operatorname{dim}(M)$ is even, then the fixed point set is not empty.

(1.2.2) If $\operatorname{dim}(M)$ is odd, then there is a circle orbit.

Note that (1.2.2) follows easily from (1.2.1) (cf. [GS], [Ro1], [Su]).

By Theorem 1.2 and the isotropy representation at an orbit of minimal dimension, one concludes the following $([\mathrm{GS}])$ :

COROLlary 1.3. Let a torus $T^{k}$ act isometrically on a closed $n$-manifold $M$ of positive sectional curvature. Then $k \leq\left[\frac{n+1}{2}\right]$ (the integer part).

c. Proof of Theorem A. The proof of Theorem A is divided into two cases depending on $\operatorname{dim}(M)$ being even or odd. Because the proofs are in both cases are almost identical (see Remark 1.5), we will only present the proof for $\operatorname{dim}(M)$ being odd (which is also the case used in this paper). 
Lemma 1.4. Theorem $A$ is true if $\phi$ is an orientation-reversing isometry.

Proof. We proceed by induction on $\operatorname{dim}(M)=2 m+1$, starting with $m=1$ and thus $k=1$ or 2 . By the classical Synge theorem, $\phi$ has a fixed point $x \in M$ and thus $\phi$ fixes $T^{k}(x)$.

Case 1. Assume that $k=1$. Without loss of generality, we may assume that $T^{1}(x)=x$. If $F$ denotes the $T^{1}$-fixed point component at $x$, then $\phi(F)=F$. Because $\operatorname{dim}(F)=1$, from the isotropy representation of $T^{1}$ and $\phi$ on the normal space of $F$ at $x$, we then see the desired result.

Case 2. Assume that $k=2$. Let $F_{\phi}$ denote the $\phi$-fixed point component at $x$. Because $T^{2}$ preserves $F_{\phi}$, the induced $T^{2}$-action on $F_{\phi}$ cannot be trivial (otherwise, the $T^{2}$-fixed point set is not empty; which is not possible). Hence, $F_{\phi}$ is also a circle $T^{2}$-orbit.

In general, let $F_{\phi}$ denote a $\phi$-fixed point component. If the effective part of the $T^{k}$-action on $F_{\phi}$ is not trivial, then $F_{\phi}$ contains a circle $T^{k}$-orbit (Theorem 1.2). Otherwise, we may assume that $F_{\phi}$ is contained in the $T^{k}$-fixed point set. We then consider the $T^{k}$-action and the $\phi$-action on the unit normal sphere to $F_{\phi}$ at $x$ (via the isotropy representation). If $F_{\phi}$ has even codimension, then we may apply the inductive assumption to conclude that $\phi$ preserves some circle $T^{k}$-orbit on the normal sphere and therefore preserves some circle $T^{k}$-orbit on $\tilde{M}$ (note that $\phi$ preserves the orientation of the subspace tangent to $F_{\phi}$, and thus must reverse the orientation on the normal space). If $F_{\phi}$ has odd codimension, and if the $T^{k}$-action on $F_{\phi}$ is not trivial, then $F_{\phi}$ contains a circle $T^{k}$-orbit (Theorem 1.2). If $F_{\phi}$ has odd codimension, and if the $T^{k}$-action on $F_{\phi}$ is trivial, then there is a $T^{k}$-fixed component, $F_{0} \supset F_{\phi}$. Let $x \in F_{\phi}$. Because $F_{0}$ has even codimension and $\phi$ preserves $F_{0}, \phi$ and $T^{k}$ act on the unit normal sphere of $F_{0}$ at $x$. Now we can apply the inductive assumption to conclude the desired result.

By Lemma 1.4, we will assume, in the rest of the proof of Theorem A, that $\phi$ is an orientation-preserving isometry.

Proof of Theorem $A$ for $k=1$. Because $\phi$ commutes with the $T^{1}$-action, $\phi$ descends to an isometry $\phi^{*}$ on the orbit space $M^{*}=M / T^{1}$ (which may not be effective). Clearly, $\phi$ preserves an orbit if and only if $\phi^{*}$ fixes its projection on $M^{*}$.

We proceed by induction on $n$, where $\operatorname{dim}(M)=2 n+1$. The case for $n=1$ is clear because $M^{*}$ is homeomorphic to either a two sphere or a two disk.

We now argue by contradiction. Assume that the displacement function, $d^{*}\left(x^{*}, \phi^{*}\right.$ $\left.\left(x^{*}\right)\right)$ achieves the positive minimum at $x^{*}$, i.e. $0<d^{*}\left(x^{*}, \phi^{*}\left(x^{*}\right)\right) \leq d^{*}\left(y^{*}, \phi^{*}\left(y^{*}\right)\right)$ for all $y^{*} \in M^{*}$. Fixing $x \in p^{-1}\left(x^{*}\right)$, let $\sigma$ denote a geodesic from $x$ to $T^{1}(\phi(x))$ such that length $(\sigma)=d^{*}\left(x^{*}, \phi^{*}\left(x^{*}\right)\right)=$ length $\left(\sigma^{*}\right)$. Because $\phi$ commutes with the $T^{1}$-action, the isotropy groups $T_{x}^{1}=T_{\phi(x)}^{1}$. By Lemma 1.1, for all $0<t<1$, the isotropy group $T_{\sigma(t)}^{1}=H$ is independent of $t$ and is contained in $T_{x}^{1}$. Let $F$ denote the $H$-fixed point component at $x$.

If $H \neq\{1\}$ is finite, then $F$ is a closed totally geodesic $(2 k+1)$-submanifold on which $T^{1} / H$ acts effectively. Because $\phi(F) \cap F \neq \emptyset, \phi$ preserves $F$. Thus we can apply the inductive assumption and conclude that $\phi^{*}$ has a fixed point in $F^{*}$, and therefore a fixed point in $M^{*}$; a contradiction.

In the rest of the proof, we consider the remaining cases that $H=\{1\}$ or $H=T^{1}$.

Case 1. Assume that $H=\{1\}$. We claim that $T^{1}(x)$ must be a principle circle 
orbit. Assuming this, then $\sigma^{*} \subset M_{0}^{*}$. Note that $M_{0}^{*}$ is an even-dimensional open manifold of positive sectional curvature. Because the $T^{1}$-action preserves the orientation on $M_{0}, M_{0}^{*}$ is orientable, and because $\phi$ is an orientation-preserving isometry, $\phi^{*}$ is an orientation-preserving isometry. Following the standard Synge-type argument as in (2.7.1), the displacement function of $\phi^{*}$ cannot achieve a (local) minimum at $x^{*}$, a contradiction.

If $T^{1}(x)$ is not a principle orbit, we will derive a contradiction as follows: For small $\delta>0$, let $x_{\delta}=\sigma(\delta)$. Then

$$
\begin{aligned}
d\left(x_{\delta}, t \circ \phi\left(x_{\delta}\right)\right) & \leq d\left(x_{\delta}, t \circ \phi(x)\right)+d\left(t \circ \phi(x), t \circ \phi\left(x_{\delta}\right)\right) \\
& =d\left(x_{\delta}, t \circ \phi(x)\right)+d\left(x, x_{\delta}\right)=d(x, t \circ \phi(x)) .
\end{aligned}
$$

Because $d^{*}\left(x^{*}, \phi^{*}\left(x^{*}\right)\right)$ is minimal, the above must be an equality, and thus $x_{\delta}, t \circ \phi(x)$ and $t \circ \phi\left(x_{\delta}\right)$ are in some minimal geodesic, say $\alpha$ from $x_{\delta}$ to $t \circ \phi(x)$ and to $t \circ \phi\left(x_{\delta}\right)$. By the same reason, $\alpha$ must be a horizontal geodesic. Because the isotropy groups at $x_{\delta}$ and $t \circ \phi\left(x_{\delta}\right)$ are trivial but not trivial at $t \circ \phi(x)$, we get a contradiction to Lemma 1.1 .

Case 2. Assume that $H=T^{1}$ and thus $T_{x}^{1}=T^{1}$. In this case, $T^{1}$ acts on $T_{x} M$ via differentials. Let $T_{x}^{\perp}(\sigma) \subset T_{x} M$ denote the orthogonal complement of $\sigma^{\prime}(0)$, and let $\psi=d \phi^{-1} \circ P_{\sigma}: T_{x}^{\perp}(\sigma) \rightarrow T_{x}^{\perp}(\sigma)$, where $P_{\sigma}$ is the parallel translation along $\sigma$ from $\sigma(0)$ to $\sigma(1)$. Then $\psi$ is a linear isometry on $T_{x}^{\perp}(\sigma)$, and we claim that there is $V \in T_{x}^{\perp}(\sigma)$ such that $\psi(V)=d t(V)$ for some $t \in T^{1}$.

Assuming the claim, we will derive a contradiction. Let $V(t)$ denote the parallel vector field along $\sigma$ with $V(0)=V$ and let $\sigma_{\epsilon}(t)=\exp _{\sigma(t)} \epsilon V(t)$ for some fixed small $\epsilon$. By the second variation formula of arc length, we have that length $\left(\sigma_{\epsilon}\right)<\operatorname{length}(\sigma)$. Put $y=\exp _{x} \epsilon V$. Then $\phi^{*}\left(y^{*}\right)=\left(\phi\left(\exp _{x} \epsilon V\right)\right)^{*}=\left(\exp _{\phi(x)} d \phi(V)\right)^{*}=\sigma(1)^{*}$. But

$$
d^{*}\left(y^{*}, \phi^{*}\left(y^{*}\right)\right) \leq \operatorname{length}\left(\sigma_{\epsilon}^{*}\right) \leq \operatorname{length}\left(\sigma_{\epsilon}\right)<\operatorname{length}(\sigma)=d^{*}\left(x^{*}, \phi^{*}\left(x^{*}\right)\right),
$$

a contradiction.

Because $\sigma(t)$ is contained in the $T^{1}$-fixed point set, the $T^{1}$-action commutes with the parallel translation $P_{\sigma}$, and therefore $\psi$ commutes with the $T^{1}$-action on $T_{x}^{\perp}(\sigma)$. Let $S^{2 n-1}$ denote the unit ball in $T_{x}^{\perp}(\sigma)$. Then $\psi$ induces an isometric action on $S^{2 n-1}$ commuting with the $T^{1}$-action. Since both $d \phi$ and the $T^{1}$-action on $S^{2 n-1}$ are orientation-preserving, by the inductive assumption, $\psi^{*}$ has a fixed point in $S^{2 n-1} / T^{1}$ and thus there is a $V \in S^{2 n-1}$ such that $\psi(V)=d t(V)$ for some $t \in T^{1}$.

Proof of Theorem $A$ for $k>1$. We first show that $\phi$ preserves some $T^{k}$-orbit. Let $T^{1} \subset T^{k}$ be any circle subgroup. By the case of $k=1$, we may assume that $\phi$ preserves $T^{1}(x)$ for some $x \in M$. Then $\phi$ preserves $T^{k}(x)$. Consequently, there is $t \in T^{k}$ such that $t \gamma(x)=x$.

If $T^{k}(x) \neq\{x\}$, let $F$ denote the $t \gamma$-fixed point component at $x$. Because the induced $T^{k}$-action on $F$ is not trivial, $F$ contains a circle $T^{k}$-orbit (Theorem 1.2).

If $T^{k}(x)=\{x\}$, then $\phi(x)=x$. From the last part of the proof of Lemma 1.4, we conclude that $\phi$ preserves a circle $T^{k}$-orbit.

Remark 1.5. The above proof can be easily modified to a proof for even dimensions; one replaces "orientation-preserving" by "orientation-reversing", and makes corresponding obvious modifications due to this change.

Remark 1.6. Using the version of the Synge theorem for an Alexandrov space of 
positive curvature $([\mathrm{Pe}])$, one may give an alternative proof of Theorem A. However, the present proof is elementary in the sense it does not require (complicated) notions such as parallel translations in Alexandrov spaces.

d. Proof of Corollary 0.1. We will prove the following more general Corollary $0.1^{\prime}$.

COROLlary 0.1'. Let $M$ be a closed manifold of positive sectional curvature on which $T^{k}$ acts isometrically. Then the subgroup of $\pi_{1}(M)$ generated by loops in a principle $T^{k}$-orbit is cyclic, $\langle\sigma\rangle$, such that for all $\gamma \in \pi_{1}(M), \sigma$ and $\gamma$ generate a cyclic group. If $\sigma \neq 1$, then $\pi_{1}(M)$ is not isomorphic to $\mathbb{Z}_{p} \oplus \mathbb{Z}_{p}$ with any prime $p$.

Proof of Corollary 0.1'. Consider the pullback torus $\tilde{T}^{k}$-action on the Riemannian universal covering space $\tilde{M}$. Let $\Gamma_{0}$ denote the subgroup of $\pi_{1}(M)$ generated by loops in some principle $T^{k}$-orbit. Then $\Gamma_{0}$ preserves all principle $\tilde{T}^{k}$-orbits and therefore all $\tilde{T}^{k}$-orbits. Because there is a circle $\tilde{T}^{k}$-orbit (Theorem 1.2), $\Gamma_{0}=\langle\sigma\rangle$ is cyclic.

For $\gamma \in \pi_{1}(M)$, assume that $\gamma$ preserves some circle $T^{k}$-orbit in $\tilde{M}$ (Theorem A). Because $\sigma$ also preserves this circle orbit, $\langle\sigma, \gamma\rangle$ is cyclic.

2. Proof of Theorem B. In spirit, the first part of the proof is similar to [Ro2]. The new ingredients in the present proof are Theorem A and Theorem 2.9.

a. Bounding fixed point components. In the proof of Theorem $\mathrm{B}$, the index bound is derived from the following fact:

LEMMA 2.1. Let $M$ be a closed $n$-manifold of positive sectional curvature on which $T^{1}$ acts isometrically. For any finite subgroup, $\mathbb{Z}_{h} \subset T^{1}$, the number of the $\mathbb{Z}_{h}$-fixed point components is bounded above by a constant depending only on $n$.

Lemma 2.1 is a consequence of a combination of the following two theorems:

THEOREM $2.2([\mathrm{Hs}])$. Let a compact abelian Lie group $G$ act effectively on a closed manifold $M$. Then

$$
\operatorname{rank}\left(H_{*}(F(G, M), \ell)\right) \leq \operatorname{rank}\left(H_{*}(M, \ell)\right),
$$

where $G=T^{k}$ and $\ell=\mathbb{Z}$ or $G=\mathbb{Z}_{p}^{k}$ and $\ell=\mathbb{Z}_{p}$.

THEOREM $2.3([\mathrm{GR}])$. Let $M^{n}$ be a closed $n$-manifold of nonnegative sectional curvature. Then the total Betti number with respect to any coefficient field $\ell$ is,

$$
B(M)=\sum_{i=0}^{n} \operatorname{rank}\left(H_{i}(M, \ell)\right) \leq b(n) .
$$

Proof of Lemma 2.1. We proceed by induction on $n$, starting with the trivial case $n=3$. Let $h=p q$ with $p$ a prime. Then

$$
\begin{array}{ll}
\text { \# } & \text { of components of } F\left(\mathbb{Z}_{p}, M\right) \\
& \leq \operatorname{rank}\left(H_{*}\left(F\left(\mathbb{Z}_{p}, M\right), \mathbb{Z}_{p}\right)\right) \\
& \leq \operatorname{rank}\left(H_{*}\left(M, \mathbb{Z}_{p}\right)\right) \quad(\text { see Theorem 2.2) } \\
& \leq b(n)
\end{array}
$$

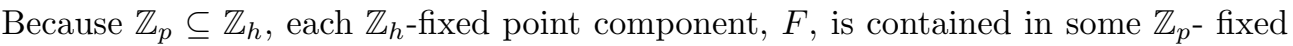


point component $E$. Because $E$ is a totally geodesic submanifold of even codimension, we can apply the inductive assumption to $\left(E,\left.T^{1}\right|_{E}\right)$, and conclude that the number of $\mathbb{Z}_{h}$-fixed point components in $E$ is bounded above by a constant $c(n)$. Then $b(n) c(n)$ gives the desired bound.

b. A special case. In this subsection, we will prove a special case of Theorem B (see Theorem 2.4). By a result in [FY] (see Theorem 2.9 below), the general case can be derived from this special case.

Let $\Gamma$ denote a group. If there is a sequence of subgroups,

$$
\Gamma=\Lambda_{0} \supset \Lambda_{1} \supset \cdots \supset \Gamma_{\ell}=\{1\}
$$

such that $\Lambda_{i+1}$ is normal in $\Lambda_{i}$ and $\Lambda_{i} / \Lambda_{i+1}$ is cyclic, we call $\left\{\Lambda_{i}\right\}$ a filtration of $\Gamma$ with polycyclicity $=\ell$. Clearly, $\Gamma$ with a filtration is a solvable group.

THEOREM 2.4. Let $M$ be a closed $n$-manifold of positive sectional curvature on which $T^{1}$ acts isometrically. If $\pi_{1}(M)$ has a filtration with polycyclicity $=\ell$, then $\pi_{1}(M)$ has a cyclic subgroup of index $\leq w(n, \ell)$, a constant depending only on $n$ and $\ell$.

As a preparation, we will prove three lemmas.

LEMMA 2.5. Let $M$ be a closed $n$-manifold of positive sectional curvature on which $T^{1}$ acts isometrically. Assume that $\pi_{1}(M)$ has a normal subgroup $\Lambda$ such that $\pi_{1}(M) / \Lambda$ is a cyclic group. If $\Lambda$ has a cyclic subgroup $C$ with index $\leq a$, then $C$ extends to a cyclic subgroup with index in $\pi_{1}(M)$ less than $c(n, a)$.

Sublemma 2.6. Lemma 2.5 is true for $n=3$.

Proof. Consider the tower of normal Riemannian covering spaces and the associated lifting circle actions:

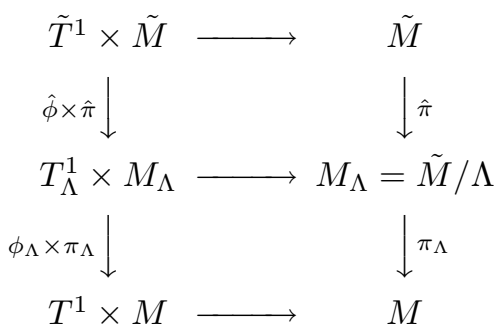

Here $\phi=\phi_{\Lambda} \circ \hat{\phi}, \pi=\pi_{\Lambda} \circ \hat{\pi}$ and $T_{\Lambda}^{1}$ denotes the lifting $T^{1}$-action on $M_{\Lambda}$.

If the $T_{\Lambda}^{1}$-action on $M_{\Lambda}$ is free, then $\Lambda \subseteq<\sigma>$ ( $\sigma$ denotes the homotopy class of a principle $T^{1}$-orbit in $M$ ) and thus $\pi_{1}(M)$ is cyclic (Corollary 0.1).

If the $T_{\Lambda}^{1}$-fixed point set is not empty, then the $\tilde{T}^{1}$-fixed point set is also not empty. Because $\tilde{M}$ is a homotopy sphere, the $\tilde{T}^{1}$-fixed point set is connected (Theorem 2.2). Because $\pi_{1}(M)$ preserves the $\tilde{F}$-fixed point set (which is a circle), $\pi_{1}(M)$ is cyclic.

If $T_{\Lambda}^{1}\left(x_{\Lambda}\right)$ is an exceptional orbit, we may assume $\mathbb{Z}_{p} \subset T_{\Lambda}^{1}$ and a $\mathbb{Z}_{p}$-fixed point component $F_{\Lambda}=T_{\Lambda}^{1}\left(x_{\Lambda}\right)$. Let $\tilde{F}=\pi_{\Lambda}^{-1}\left(\hat{F}_{\Lambda}\right)$, and let $H$ denote the subgroup of $\pi_{1}(M)$ 
which preserves $\tilde{F}$. Then $H$ is cyclic (because $\tilde{F}$ is a circle). Moreover,

$$
\begin{aligned}
{\left[\pi_{1}(M): H\right] } & \leq \# \text { components of } F\left(\mathbb{Z}_{p}, M_{\Lambda}\right) \\
& \leq \operatorname{rank}\left(H_{*}\left(F\left(\mathbb{Z}_{p}, M_{\Lambda}\right), \mathbb{Z}_{p}\right)\right. \\
& \leq \operatorname{rank}\left(H_{*}\left(M_{\Lambda}, \mathbb{Z}_{p}\right)\right) \leq b(3) .
\end{aligned}
$$

Lemma 2.8. Let $\Lambda_{1}, \Lambda_{2}$ be two subgroups of a finite group $\Gamma$. Then

$$
\left[\Lambda_{2}: \Lambda_{1} \cap \Lambda_{2}\right] \leq\left[\Gamma: \Lambda_{1}\right]
$$

We omit the proof here because it is straightforward to check.

Proof of Lemma 2.5. We proceed by induction on $n$ starting with $n=3$ (Sublemma 2.6).

Let $C$ denote a cyclic subgroup of $\Lambda$ with index $\leq a$. Consider the tower of normal Riemannian covering spaces and the lifting circle actions as in (2.7).

Case 1. Assume that $C$ is not contained in $\langle\sigma\rangle$. This implies that $C$ does not preserve any principle $T^{1}$-orbit in $\tilde{M}$. But $C$ preserves some circle $T^{1}$-orbit (Theorem A), whose projection in $M_{\Lambda}$ must be an exceptional $T_{\Lambda}^{1}$-orbit. Let $H \subset T_{\Lambda}^{1}$ denote the isotropy group of this exceptional orbit, and let $F_{\Lambda}$ denote the $H$-fixed point component. Let $\tilde{F}=\hat{\pi}^{-1}\left(F_{\Lambda}\right)$, and let $\Lambda_{0}$ denote the subgroup of $\pi_{1}(M)$ which preserves $\tilde{F}$. Clearly, $C \subseteq \Lambda_{0}$. Note that $\tilde{F}$ is a $T^{1}$-invariant closed totally geodesic submanifold and $\Lambda_{0}$ has a normal subgroup $\Lambda_{0} \cap \Lambda$ such that the quotient group is cyclic. If $\tilde{F}$ is simply connected, then we can apply the inductive assumption on $\left(\tilde{F} / \Lambda_{0}, T_{0}^{1}\right)$ to conclude that $C$ extends to a cyclic subgroup $C_{0}$ of $\Lambda_{0}$ with index $\leq w(n, a)$. If $\tilde{F}$ is not simply connected, it is easy to see that one can still apply the inductive assumption, because $\pi_{1}\left(\tilde{F} / \Lambda_{0}\right)$ satisfies the following exact sequence,

$$
\{1\} \rightarrow \pi_{1}(\tilde{F}) \rightarrow \pi_{1}\left(\tilde{F} / \Lambda_{0}\right) \stackrel{f}{\rightarrow} \Lambda_{0} \rightarrow\{1\} .
$$

Let $C^{\prime}$ denote a cyclic subgroup in $\pi_{1}\left(\tilde{F} / \Lambda_{0}\right)$ such that $f\left(C^{\prime}\right)=C$. By the inductive assumption, $C^{\prime}$ extends to a cyclic subgroup $C_{0}^{\prime}$ of $\pi_{1}\left(\tilde{F} / \Lambda_{0}\right)$ with index bounded by $w\left(n, a,\left|\pi_{1}(\tilde{F})\right|\right)$. Then $C_{0}=f\left(C_{0}^{\prime}\right)$ is a cyclic subgroup of $\Lambda_{0}$ with index bounded by $w(n, a)$. Then $\left[\pi_{1}(M): C_{0}\right]=\left[\pi_{1}(M): \Lambda_{0}\right] \cdot\left[\Lambda_{0}, C_{0}\right] \leq w(n, a)\left[\pi_{1}(M): \Lambda_{0}\right]$.

Because $\pi_{1}(M) / \Lambda$ is the covering transformation group commuting with the $T_{\Lambda^{-}}^{1}$ action,

$$
\text { \# of components of } \begin{aligned}
\pi_{\Lambda}^{-1}(\tilde{F} / \Lambda) & \leq \# \text { of components of } F\left(H, M_{\Lambda}\right) \\
& \leq c(n, a)(\text { Lemma 2.1). }
\end{aligned}
$$

Note that for $\gamma_{1}, \gamma_{2} \in \pi_{1}(M), \gamma_{1} \Lambda_{0}=\gamma_{2} \Lambda$ if and only if $\gamma_{1}(\tilde{F})=\gamma_{2}(\tilde{F})$. Hence,

$$
\begin{aligned}
{\left[\pi_{1}(M): \Lambda_{0}\right] } & =\# \text { of components of } \pi^{-1}(\pi(\tilde{F})) \\
& =\# \text { of components of } \hat{\pi}^{-1}\left(F_{\Lambda}\right) \cdot \# \text { of components of } \pi_{\Lambda}^{-1}\left(\tilde{F} / \Lambda_{0}\right) \\
& \leq\left[\Lambda, \Lambda_{0} \cap \Lambda\right] \cdot c(n, a) \\
& \leq[\Lambda, C] \cdot c(n, a) \quad(\text { see Lemma 2.8) } \\
& \leq a \cdot c(n, a) .
\end{aligned}
$$


Case 2. Assume that $C$ is a subgroup of $\langle\sigma\rangle$. If $\Lambda$ is not contained in $\langle\sigma\rangle$, then the $T_{\Lambda}^{1}$-action on $M_{\Lambda}$ has a finite isotropy group. It is straightforward to check that the argument in Case 1 goes through with the obvious minor modification.

Case 3. Assume that $\Lambda \subseteq\langle\sigma\rangle$. In this case, by Theorem A, $\pi_{1}(M) / \Lambda=\langle\alpha\rangle$ preserves some circle orbit $T^{1}(x), x \in \tilde{M}$. Because $\Lambda$ preserves every $T^{1}$-orbit, $\pi_{1}(M)$ is cyclic.

Proof of Theorem 2.4. Consider a filtration of $\pi_{1}(M)$ :

$$
\pi_{1}(M)=\Lambda_{0} \supset \Lambda_{1} \cdots \supset \Lambda_{k}=\{1\} .
$$

Let $M_{i}=\tilde{M} / \Lambda_{i}$, on which there is an induced $T^{1}$-action.

We first consider $\left(M_{k-2}, T^{1}\right)$. By Lemma $2.5, \Lambda_{k-1}$ extends to a cyclic subgroup $C_{k-2}$ of $\Lambda_{k-2}$ with index less than $a_{k-2}(n)$. We then consider $\left(M_{k-3}, T^{1}\right)$. Again by Lemma 2.5, $C_{k-2}$ extends to a cyclic subgroup $C_{k-3}$ with index less than $a_{k-1}(n)$. Repeating this a number of times, we then get the desired result.

c. Proof of Corollary 0.3. To derive Corollary 0.3 from Theorem 2.4, the following result is crucial.

TheOREm 2.9 ([FY]). Given $n$, there are constants, $\epsilon=\epsilon(n), w=w(n)$, such that if a closed $n$-manifold $M$ admits a metric with $\sec _{M^{n}} \cdot(\operatorname{diam}(M))^{2} \geq-\epsilon$, then the fundamental group of $M$ has a normal solvable subgroup $\Gamma$ satisfying the following conditions:

(2.9.1) $\Gamma$ has polycyclicity at most $n$.

(2.9.2) $\left[\pi_{1}(M): \Gamma\right] \leq w_{n}$.

We also need the following algebraic lemma.

Lemma 2.10. Let $C$ be a cyclic subgroup of a finite group $\Gamma$ with index a. Then $C$ contains a subgroup $C_{0}$ such that $C_{0}$ is normal in $\Gamma$ with index at most $a^{a+1}$.

Proof. Let

$$
C_{0}=\bigcap_{\gamma \in \Gamma} \gamma^{-1} C \gamma
$$

Then $C_{0} \subseteq C$ is a normal subgroup of $\Gamma$ (note that $C_{0}$ may be trivial).

We first claim that $C$ has at most $a$ conjugate classes, i.e. the set $\left\{\gamma^{-1} C \gamma, \gamma \in \Gamma\right\}$ contains at most $a$ elements. By Lemma 2.8,

$$
\left[C: C \cap \gamma^{-1} C \gamma\right]=\left[\gamma^{-1} C \gamma: C \cap \gamma^{-1} C \gamma\right] \leq[\Gamma: C] \leq a .
$$

The above implies the claim because $C$, a cyclic group, can contain at most $a$ subgroups with index less than $a$. Let $\left\{\gamma^{-1} C \gamma, \gamma \in \Gamma\right\}=\left\{\gamma_{i}^{-1} C \gamma_{i}, 0 \leq i \leq s \leq a\right\}$, and let $C_{i}=\gamma_{i}^{-1} C \gamma_{i}$. By repeatedly applying Lemma 3 , we derive

$$
\begin{aligned}
{\left[\Gamma: C_{0}\right] } & =[\Gamma: C] \cdot\left[C: C \cap C_{1}\right] \cdots\left[C \cap C_{1} \cap \cdots \cap C_{s-1}: C_{0}\right] \\
& \leq a \cdots a=a^{s+1} \leq a^{a+1}
\end{aligned}
$$

LEMma 2.11. For each $k$, any torsion subgroup of the special linear group $S L(k, \mathbb{Z})$ has order at most $3^{k^{2}}$. 
For a proof, see Lemma 3.5 of [FR3].

Proof of Corollary 0.3. Let $\operatorname{ker}(\rho)$ denote the kernel of the holonomy representation, $\rho: \pi_{1}(M) \rightarrow \operatorname{Aut}\left(T^{k}\right) \cong \mathrm{SL}(k, \mathbb{Z})$. By Lemma 2.11 and Corollary 1.3, $\left[\pi_{1}(M): \operatorname{ker}(\rho)\right] \leq 3^{k^{2}} \leq 3^{\left[\frac{n+1}{2}\right]^{2}}$. Because $\operatorname{ker}(\rho)$ commutes with the $T^{k}$-action on $\tilde{M}$, we can apply Theorem 2.9 to $\tilde{M} / \operatorname{ker}(\rho)$ and conclude that $\operatorname{ker}(\rho)$ has a normal solvable subgroup $\Gamma_{1}$ with polycyclicity $\leq n$ such that $\left[\operatorname{ker}(\rho): \Gamma_{1}\right] \leq w_{n}$. Let $T_{1}^{k}$ denote the descending $T^{k}$ which acts on $\tilde{M} / \Gamma_{1}$. Taking any $T^{1} \subseteq T_{1}^{k}$ and applying Theorem 2.4 to $\left(\tilde{M} / \Gamma_{1}, T^{1}\right)$, we conclude that $\Gamma_{1}$ has a cyclic subgroup $C$ of index $\leq c(n)$. Then

$$
\left[\pi_{1}(M): C\right]=\left[\pi_{1}(M): \operatorname{ker}(\rho)\right] \cdot\left[\operatorname{ker}(\rho): \Gamma_{1}\right] \cdot\left[\Gamma_{1}: C\right] \leq 3^{\left[\frac{n+1}{2}\right]^{2}} \cdot w_{n} \cdot c(n)=d(n) .
$$

By Lemma 2.10, $\pi_{1}(M)$ has a normal cyclic subgroup of index $\leq d^{d+1}$.

d. Proof of Theorem B. In the proof of Corollary 0.3, we first show the existence of a cyclic subgroup of bounded index. This proof can be easily modified to a proof of Theorem B, in the presence of a nontrivial maximal normal cyclic subgroup.

Proof of Theorem B. Let $\langle\alpha\rangle$ denote a maximal normal subgroup of $\pi_{1}(M)$. If $\langle\alpha\rangle=\{1\}$, then $\pi_{1}(M)$ contains no normal cyclic subgroup. In this case, we can apply Corollary 0.3. Hence, in the rest of the proof, we may assume that $\langle\alpha\rangle \neq\{1\}$. By Corollary $0.1,\langle\alpha, \sigma\rangle$ generates a normal subgroup of $\pi_{1}(M)$ and therefore the maximality implies that $\langle\sigma\rangle \subseteq\langle\alpha\rangle$ (note that $\langle\sigma\rangle$ can be trivial).

Case 1. Assume that $\langle\sigma\rangle=\langle\alpha\rangle$. By Corollary $0.3, \pi_{1}(M)$ has a normal cyclic subgroup $\langle\beta\rangle$ of index $\leq w(n)$. By Corollary $0.1,\langle\beta, \sigma\rangle$ is also a normal cyclic subgroup of $\pi_{1}(M)$. The maximality of $\langle\alpha\rangle$ then implies that $\langle\alpha\rangle=\langle\beta\rangle$.

Case 2. Assume that $\langle\sigma\rangle \subsetneq\langle\alpha\rangle$. We proceed by induction on $\operatorname{dim}(M)=n$, starting with the trivial case $n=3$ (see Lemma 2.5).

Consider the tower of normal covering spaces with the induced $\pi_{1}$-invariant isometric $T^{k}$-action:



Assume that $\alpha$ preserves some orbit $T^{k}(x), x \in \tilde{M}$ (Corollary 0.1). Let $t \in T^{k}$ such that $t \circ \alpha(x)=x$, and let $\tilde{F}_{\alpha}$ denote the $t \circ \alpha$-fixed point component at $x$. Let $H_{\alpha}$ denote the subgroup of $\pi_{1}(M)$ which preserves $\tilde{F}_{\alpha}$. Then $\alpha \in H_{\alpha}$, and thus $\langle\alpha\rangle$ is also a maximal normal subgroup of $H_{\alpha}$. Because $\tilde{F}_{\alpha}$ contains no principle $T^{k}$-orbit, $\tilde{F}_{\alpha}$ is a closed $T^{k}$-invariant totally geodesic submanifold of even codimension, and we can apply the inductive assumption to conclude that the index of $\langle\alpha\rangle$ in $H_{\alpha}$ is $\leq a(n)$.

It remains to bound $\left[\pi_{1}(M): H_{\alpha}\right]$. Because $\langle\sigma\rangle$ is normal in $\pi_{1}(M), \gamma\left(\tilde{F}_{\alpha}\right)$ is 
a $t \circ \alpha$-fixed point component. Then

$$
\begin{aligned}
{\left[\pi_{1}(M): H_{\alpha}\right] } & =\#\left\{\gamma\left(\tilde{F}_{\alpha}\right), \gamma \in \pi_{1}(M)\right\} \\
& \leq \#\{\text { components of } F(t \circ \alpha, \tilde{M})\} \\
& \leq c(n) . \quad(\text { Lemma 2.1) }
\end{aligned}
$$

\section{Proof of Theorems $\mathrm{C}$ and $\mathrm{D}$.}

a. Proof of Theorem C. An isotropy group $H$ is called local minimal if there is an $H$-fixed point whose neighborhood contains no isotropy group other than $H$.

LEMMA 3.1. Let $T^{k}$ act on a manifold $M$.

(3.1.1) A minimal isotropy group is isomorphic to either $T^{1}$ or $\mathbb{Z}_{h}$.

(3.1.2) There is no finite isotropy group if and only if every isotropy group is connected.

Proof. (3.1.1) Assume $x \in M$ such that $1 \neq T_{x}^{k}$ is a locally minimal isotropy group. Let $F$ denote a primary component of $F\left(T_{x}^{k}, M\right)$. The minimality implies that $T_{x}^{k}$ acts freely on the unit normal sphere $S_{x}^{\perp} \subset T_{x} M$. A priori, $T_{x}^{k} \cong T^{\ell} \times A$, where $A$ is a finite abelian group. Then either $\ell=0$ or $A=1$, because otherwise one concludes a noncyclic abelian group acting freely a sphere, a contradiction to Corollary 0.3.

(3.1.2) It suffices to prove the necessity of (3.1.2). We argue by contradiction. Assume $x \in M$ such that the isotropy group at $x$ is $T_{x}^{k}=T^{\ell} \times A$, where $A \neq 1$ is a finite abelian group. Without loss of generality, we may assume that $T_{x}^{k}=T^{\ell} \times A$ is minimal, i.e. it contains no proper isotropy subgroup of the form $T^{\ell} \times B$.

Let $F$ denote the $T_{x}^{k}$-fixed point component at $x$. Then $T_{x}^{k}$ acts linearly on the normal space, $T_{x}^{\perp} F$, via the differentials. It is easy to see that if $T_{x}^{k}$ has a finite isotropy group at $u$ in the unit disk $D^{\perp} \subset T_{x}^{\perp} F$, then $T_{\exp _{x} \epsilon u}^{k}$ is finite for $\epsilon$ small. Note that $\operatorname{dim}\left(D^{\perp}\right)$ is even and the $T_{x}^{k}$-action has no fixed point in $\partial D^{\perp}$ (these two properties will be used below).

From the above, it suffices to show that $T_{x}^{k}=T^{\ell} \times A$ has a finite isotropy group on $\partial D^{\perp}$. We proceed by induction on $\ell$. For $\ell=1$ and $1 \neq \alpha \in A$, by Theorem 2.1 we can assume that $\alpha$ preserves some circle orbit $T^{1}(v), v \in \partial D^{\perp}$. If $T^{1}(v) \neq v$, i.e. $T^{1}(v)$ is a circle, then the isotropy group $\left(T^{1} \times A\right)_{v}$ of the $\left(T^{1} \times A\right)$-action on $\partial D^{\perp}$ is finite (from the earlier discussion, the proof is then complete). If $T^{1}(v)=v$, then $\alpha(v)=\alpha\left(T^{1}(v)\right)=T^{1}(v)=v$, and this implies that $\left(T^{1} \times A\right)_{v}=T^{1} \times B$ with $\alpha \in B$. But $\left(T^{1} \times A\right)_{v} \subsetneq T^{1} \times A$ because $T_{x}^{k}$ has no fixed point in $\partial D^{\perp}$. Note that $T_{\exp _{x} \epsilon v}^{k} \cong\left(T^{1} \times A\right)_{v} \subsetneq T_{x}^{k}$, and thus we obtain a contradiction to the minimality of $T_{x}^{k}$.

For $\ell>1$, let $T^{\ell-1} \subset T^{\ell}$ such that $T^{\ell-1}$ has no fixed point in $\partial D^{\perp}$, and let $T^{\ell}=T^{\ell-1} \times T^{1}$. Applying the inductive assumption on $\left(\partial D^{\perp}, T^{\ell-1} \times A\right)$, there is $v \in \partial D^{\perp}$ such that $1 \neq\left(T^{\ell-1} \times A\right)_{v}$ is finite. If $T^{1}$ does not fix $\left(T^{\ell-1} \times A\right)(v)$, then $\left(T^{\ell} \times A\right)_{v}$ is finite (the proof is then complete). Otherwise, $\left(T^{\ell} \times A\right)_{v} \cong T^{1} \times B$. From the case $\ell=1$, we conclude that the $\left(T^{1} \times B\right)$-action on $\partial D^{\perp}$ has a finite isotropy subgroup at a point $u$ near $v$, and therefore the $\left(T^{\ell} \times A\right)$-action on $\partial D^{\perp}$ has a finite isotropy group at $u$.

By Theorem A, we observe the following: each element $\gamma \in \pi_{1}(M, x)$ is homotopy 
equivalent to $\eta^{-1} \gamma_{y} \eta$, where $\eta$ is a horizontal path from $x$ to $y$ (i.e. $\eta(t)$ meets transversal to $\left.T^{k}(\eta(t))\right)$ and $\gamma_{y}$ is a loop in $T^{k}(y)$ (whose 'lifting' is preserved by $\gamma$ ). In the following discussion, for the sake of simple notation, we will omit a reference point $x$ and thus use $\gamma_{y}$ to represent $\gamma$.

Proof of Theorem C. Assume there is no finite isotropy group. By Lemma 3.1, every isotropy group is connected. We will show that every element $\gamma \in \pi_{1}(M)$ is homotopy equivalent to a loop in a principle $T^{k}$-orbit (by Corollary 0.1 , this implies the desired result).

For $x \in M$, let $U=T^{k} \times_{T_{x}^{k}} D^{\perp}$ denote a tube around the orbit $T^{k}(x)$, where the isotropy group at $x, T_{x}^{k}$ acts on the normal unit sphere via the isotropy representation $([\mathrm{Br}])$. Let $p_{t}: U \rightarrow T^{k}(x)$ denote the radial projection from $U_{t}=T^{k} \times_{T_{x}^{k}} D^{\perp}(t)$, $0 \leq t \leq 1$. Let $y \in U$ such that $T^{k}(y)$ is a principle $T^{k}$-orbit and the minimal geodesic $\alpha$ from $y$ to $x$ is orthogonal to $T^{k}(x)$. Let $\Gamma_{y}$ denote the subgroup of $\pi_{1}(M, x)$ generated by loops in $\alpha^{-1} \gamma \alpha$, where $\gamma$ is a loop in $T^{k}(y)$ at $y$. Then $p$ induces an injection $p_{*}: \Gamma_{y} \rightarrow \Gamma_{x}$.

When restricting to $T^{k}(y)$, the map $p$ coincides with the quotient map, $T^{k}(y) \rightarrow$ $T^{k}(y) / T_{x}^{k}=T^{k}(x)$. Because $T_{x}^{k}$ is connected, by the homotopy lifting property (p.91, $[\mathrm{Br}]$ ) we see that $p_{*}$ is surjective and thus isomorphic. Then the continuity of the deformation $p_{t}(0 \leq t \leq 1)$ implies that $\Gamma_{y}=\Gamma_{x}$. Because $\Gamma_{y}$ is cyclic (Corollary 0.1) and because $\Gamma_{y}$ is independent of $y, \pi_{1}(M) \cong \Gamma_{y}$ is cyclic.

b. Proof of Theorem D. We first observe a consequence of the following Synge-type result:

THEOREM $3.2([\mathrm{FR}])$. Let $M$ be a closed $n$-manifold of positive sectional curvature. If $N_{1}$ and $N_{2}$ are two closed totally geodesic submanifolds such that

$$
\operatorname{dim}\left(N_{1}\right)+\operatorname{dim}\left(N_{2}\right) \geq n
$$

then $N_{1} \cap N_{2} \neq \emptyset$.

Corollary 3.3. Let $M$ be a closed $(2 n+1)$-manifold of positive sectional curvature. If $N$ is a totally geodesic $m$-submanifold of positive sectional curvature with $m>n$, then the inclusion, $N \hookrightarrow M$, induces an onto map on the fundamental groups.

Proof. Let $\pi: \tilde{M} \rightarrow M$ denote the Riemannian universal covering, and let $\tilde{N}$ denote a component of $\pi^{-1}(N)$. Because each component of $\pi^{-1}(N)$ is a closed totally geodesic submanifold of $\tilde{M}$, by Theorem $3.2 \tilde{N}=\pi^{-1}(N)$. This implies that the inclusion, $N \hookrightarrow M$, induces an onto map on the fundamental groups.

Proof of Theorem D. By Theorem C, we may assume a maximal finite isotropy group $H \neq 1$, i.e. $H$ is not a proper subgroup of any finite isotropy group. Let $F$ denote an $H$-fixed point component containing a point whose isotropy group is $H$. Because the induced $T^{k} / H$-action on $F$ has no finite isotropy group, $\pi_{1}(F)$ is cyclic (Theorem C). Because $T^{k} / H$ acts effectively on $F, \operatorname{dim}(F) \geq \frac{n+1}{2}$ (Corollary 1.3). This implies that the inclusion, $F \hookrightarrow M$, induces an onto map on the fundamental groups (Corollary 3.3). 


\section{REFERENCES}

[Ba] Y. Bazak̀ın, A manifold with positive sectional curvature and fundamental group $\mathbb{Z}_{3} \oplus \mathbb{Z}_{3}$, Sibirsk. Mat. Zh., 40 (1999), pp. 994-996.

[Bon] O. Bonnet, Sur quelques propriétés des lignes géodésiques, C. R. Acad/Sc. Paris, 40 (1855), pp. 1311-1313.

[Br] G. BRedon, Introduction to compact transformation groups, Academic Press, 48 (1972).

[CG1] J. Cheeger; M. Gromov, Collapsing Riemannian manifolds while keeping their curvature bounded I, J. Diff. Geom.,, 23 (1986), pp. 309-364.

[CG2] J. Cheeger; M. Gromov, Collapsing Riemannian manifolds while keeping their curvature bounded II, J. Diff. Geom.,, 32 (1990), pp. 269-298.

[Ch] S.S. Chern, Proc. of US-Japan Seminar in Differential Geometry, Kyoto, Japan (1965).

[DM] J. F. Davis; R. J. Milgram, A survey of the spherical spaces form problem, Math. reports volume, part 2 Harwood Academic Publishers, 2.

[FMR] F. FAng; S. Mendonca; X. Rong, A connectedness principle in the geometry of positive curvature, Comm. Anal. Geom., 13 (2005), pp. 479-501.

[FR1] F. FAnG; X. Rong, Positively curved manifolds of maximal discrete symmetry rank, Amer. J. Math, 126 (2004), pp. 227-245.

[FR2] F. FANG; X. Rong, Homeomorphism classification of positively curved manifolds of almost maximal symmetry rank, Math. Ann., 332 (2005), pp. 81-101.

[FR3] F. FAnG; X. Rong, The twisted second Betti number and convergence of collapsing Riemannian manifolds, Invent. Math., 150 (2002), pp. 61-109.

[FR4] F. FAnG; X. Rong, Collapsed 5-manifolds with pinched positive sectional curvature, to appear.

[Fr] T. Frankel, Manifolds of positive curvature, Pacific J. Math., 11 (1961), pp. 165-174.

[FRW] P. Frank, X. RONG, Y. WANG, Fundamental groups of positively curved manifolds with symmetry, preprint.

[FY] K. FukaYa; T. Yamaguchi, The fundamental groups of almost nonnegatively curved manifolds, Ann. of Math., 136 (1992), pp. 253-333.

[Gr] M. Gromov, Curvature, diameter and Betti numbers, Comm. Math. Helv., 56 (1981), pp. 179-195.

[Gro] K. Grove, Geometry of, and via symmetries, Univ. Lecture Ser., Amer. Math. Soc., Providence, RT, 27 (2002), pp. 31-53.

[GS] K. Grove; C. Searle, Positively curved manifolds with maximal symmetry-rank, J. Pure Appl. Alg., 91 (1994), pp. 137-142.

[GSh] K. Grove; K. Shankar, Rank two fundamental groups of positively curved manifolds, J. Geom. Anal., 4 (2000), pp. 679-682.

[GSZ] K. Grove; K. Shankar; W. Ziller, On the cohomogeneity and symmetries of Eschenburg and Basaikin spaces, In preparation.

[Hs] W. HsiAnG, Cohomology theory of topological transformation groups, Ergebnisse der Mathematik und inere Grenzgebiete, 85 (1975).

[Kl] B. KLEINER, Riemannian four-manifolds with nonnegative curvature and continuous symmetry, Thesis, UC at Berkeley. (1989).

[My] S. Myers, Riemannian manifolds with positive mean curvature, Duke Math. J., 8 (1941), pp. 401-404.

[Pe] A. Petrunin, Parallel transportation for Alexandrov space with curvature bounded below, Geom. Funct. Anal., 8 (1998), pp. 123-148.

[Ro1] X. Rong, On the fundamental groups of compact manifolds of positive sectional curvature, Ann. of Math., 143 (1996), pp. 397-411.

[Ro2] X. Rong, Positive curvature, local and global symmetry, and fundamental groups, Amer. J. Math., 121 (1999), pp. 931-943.

[Ro3] X. Rong, Collapsed manifolds with pinched positive sectional curvature, J. Diff. Geom., 51 (1999), pp. 335-358.

[Ro4] X. Rong, Positively curved manifolds with almost maximal symmetry rank, Geom. Dedicata, 59 (2002), pp. 157-182.

[RW1] X. Rong; Y. WANG, Fundamental groups of closed manifolds with positive curvature and torus actions, Geometriae Dedicata, 113 (2005), pp. 165-184. 
[RW2] X. RonG; Y. WANG, Classification of fundamental groups of positively curved $n$-manifolds with symmetry rank $>\frac{n}{6}$, preprint.

[Sh] K. Shankar, On fundamental groups of positively curved manifolds, J. Differential Geom, 49 (1998), pp. 179-182.

[Su] K. Sugahara, The isometry group of and the diameter of a Riemannian manifold with positive curvature, Math. Japan, 27 (1982), pp. 631-634.

[Sy] J. Synge, On the connectivity of spaces of positive curvature, Quarterly J. Math. Oxford, 7 (1936), pp. 316-320.

[Wi] B. Wilking, Torus actions on manifolds of positive sectional curvature, Acta Math (2003), pp. 259-297.

[Wo] J. A. Wolf, The spaces of constant curvature, McGraw-Hill series (1976).

[Ya] S-T. YAu, Problem section, Seminar on differential geometry, Annals of Math. Studies, Princeton University Press, 102 (1982), pp. 669-706. 
X. RONG 